\title{
Balkanologie
}

Balkanologie Revue d'études pluridisciplinaires

Vol. X, $n^{\circ} 1-2 \mid 2008$

Volume $X$ Numéro 1-2

\section{Les Balkans dans le système spatial danubien : entre intégration européenne et différenciation régionale}

\section{Veselin Siarov}

\section{OpenEdition}

Journals

Édition électronique

URL : http://journals.openedition.org/balkanologie/406

DOI : 10.4000/balkanologie.406

ISSN : 1965-0582

Éditeur

Association française d'études sur les Balkans (Afebalk)

\section{Référence électronique}

Veselin Siarov, «Les Balkans dans le système spatial danubien : entre intégration européenne et différenciation régionale », Balkanologie [En ligne], Vol. X, n 1-2 | 2008, mis en ligne le 03 juin 2008, consulté le 17 décembre 2020. URL : http://journals.openedition.org/balkanologie/406 ; DOI : https:// doi.org/10.4000/balkanologie.406

Ce document a été généré automatiquement le 17 décembre 2020.

(c) Tous droits réservés 


\title{
Les Balkans dans le système spatial danubien : entre intégration européenne et différenciation régionale
}

\author{
Veselin Siarov
}

\section{Réflexion sur un système spatial hétérogène et complexe, inégalement intégré dans l'espace ${ }^{1}$}

\footnotetext{
La position géographique du système spatial danubien ${ }^{2}$, constitue depuis toujours un facteur crucial pour le développement global de l'Europe centrale (Figures 1a et 1b). Cette situation a fait du fleuve et de son système de transport un fil directeur pour la structuration polycentrique des échanges et des transports dans cette partie de l'Europe, constituant ainsi un corridor multimodal prioritaire et un axe symbolique pour les trois derniers élargissements européens. Cependant, plus de quinze ans après le début de la transition engagée en 1989, il est toujours aussi difficile d'estimer l'ampleur des phénomènes observés dans la partie centrale et orientale du bassin, ainsi que de localiser avec précision l'évolution et la dynamique de différenciation de ce nouvel espace européen.
} 
Figure 1a. Le bassin du Danube et du Rhin au sein de l'espace européen élargi*

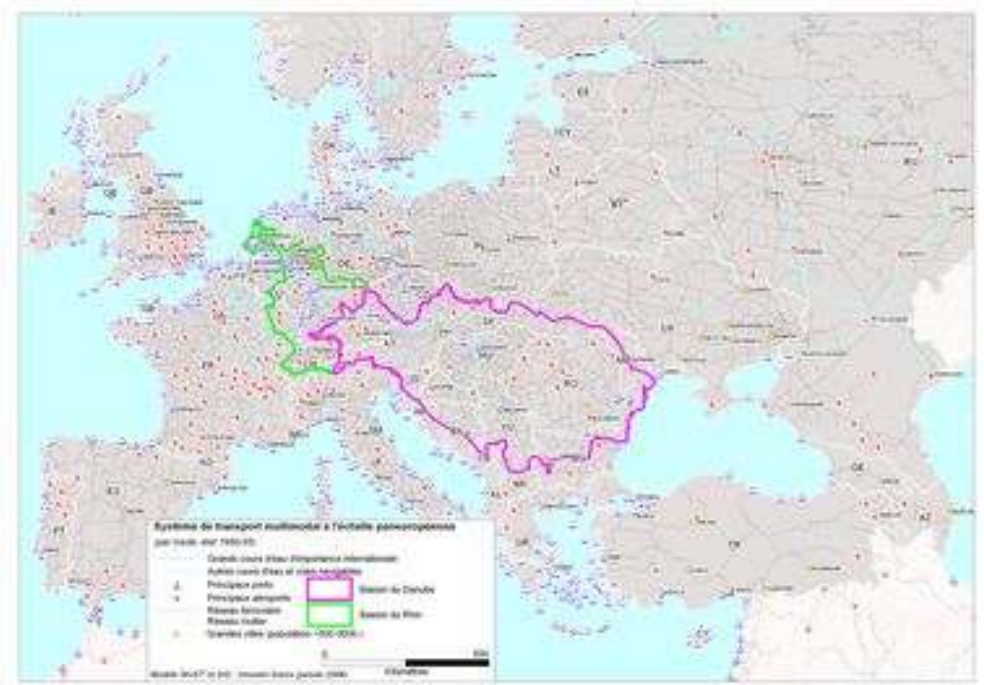

Figure 1b. Carte physique du système danubien (voies navigables et infrastructures de transport intégrées par son bassin versant)

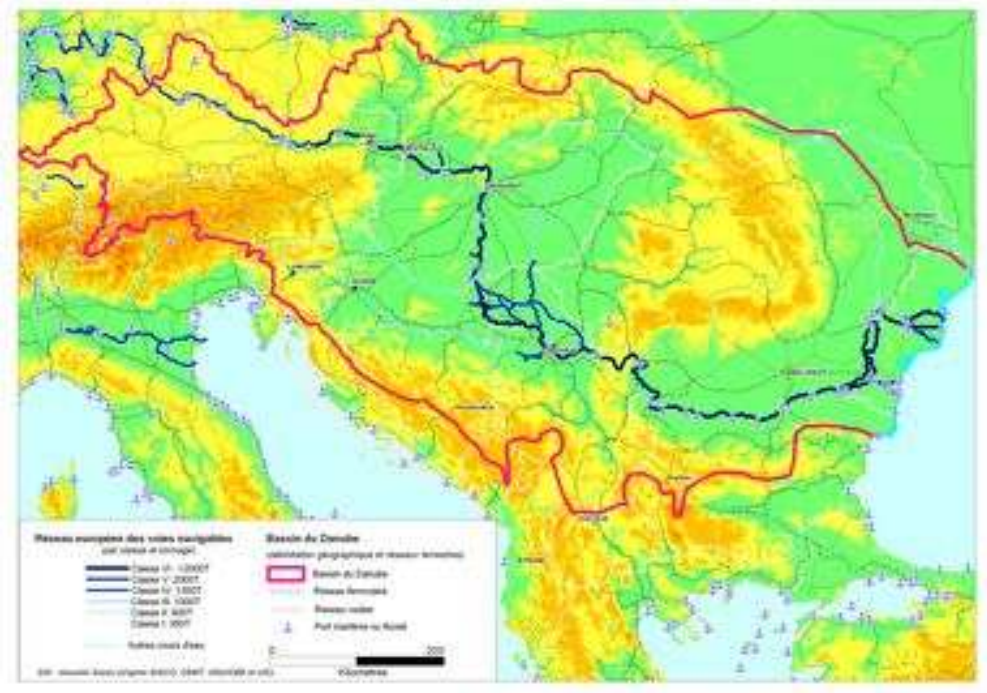

(*) Réseaux et caractéristiques de transport utilisés pour la mise au point (calibrage) du modèle spatial au sein du module des infrastructures de transport, européennes et trans-danubiennes, période 1990-1995.

La présence persistante de disparités économiques ou concernant les infrastructures de transport, à différentes échelles révélée après l'intégration d'une partie importante des pays riverains impose maintenant plus que jamais le besoin de consolider les moyens et les outils de prospective territoriale, dans la région la plus instable d'Europe centrale et orientale. En effet, l'espace danubien est partagé actuellement par une douzaine de pays très hétérogènes. L'extrémité ouest du bassin intègre des territoires occidentaux et prospères (Allemagne, Suisse, Autriche),tandis que les parties centrale et orientale sont composées soit par des ensembles territoriaux récemment intégrés dans l'Union européenne (République Tchèque, Slovaquie, Hongrie et Slovénie en 2004; Bulgarie et Roumanie en 2007),soit par despays tournés vers l'UE et placés à des degrés divers dans le processus d'intégration (Croatie ${ }^{3}$, Serbie, Bosnie-Herzégovine, Moldavie et Ukraine). 
Cette hétérogénéité territoriale délicate à cerner à cause de l'indigence de statistiques harmonisées, régionalisées (données socio-économique par NUTS ${ }^{4}$ ) et applicables aussi bien à l'échelle des pays danubiens communautaires qu'à celle des pays tiers des Balkans, complique davantage la problématique étudiée, et rend particulièrement difficile toute approche quantitative classique, à vocation supranationale ou interrégionale. Considérée à juste titre comme un véritable obstacle pour toute étude ou recherche menée dans le bassin oriental du Danube et surtout dans les Balkans, cette complexité à la fois institutionnelle et opérationnelle, est susceptible de constituer également dans le contexte concret de ce travail, l'argument générateur pour la réalisation de cette investigation expérimentale.

4 L'objet essentiel de cet article est de présenter notamment de façon illustrative, comparative et non exhaustive, quelques caractéristiques, permettant d'identifier, au moins globalement ${ }^{5}$, un certain nombre de différenciations ou de discontinuités microet macro-spatiales du système danubien. L'approche méthodologique mise au point pour la réalisation de ce travail de recherche résulte d'une recherche personnelle en cours de formalisation, qui tente de recenser et de transposer par le biais du modèle et $\mathrm{du} \mathrm{SIG}^{6}$,'évolution spatio-temporelle des principales composantes danubiennes, illustrées ici par l'organisation multi-scalaire des infrastructures multimodales (ex. réseaux de transport et terminaux), des populations et des richesses économiques ${ }^{7}$, harmonisées et compilées par le modèle.

5 Résultant des besoins réels de prospective territoriale dans cette partie de l'Europe, cette nouvelle technique est en mesure de constituer un outil puissant d'aménagement, de planification et de prévision spatio-temporelle, applicable à des échelles géographiques et opérationnelles dépassant largement le contexte du bassin et des pays danubiens. L'effet complémentaire et fonctionnel de cette recherche est d'accompagner notamment les processus nationaux d'intégration et de cohésion européennes mais également les aménagements communautaires prévisionnels ou en cours de réalisation (Tableau 1 et Figures $2 \mathrm{a}$ à $2 \mathrm{c}$ ), afin de :

- valider ou (ré)ajuster, si besoin, certains champs d'intervention politique ou d'actions prioritaires $^{8}$;

- vérifier la consistance des objectifs politiques en matière d'aménagement et d'infrastructure à moyen et à long terme, ainsi que celle des applications concrètes prioritaires engagées ;

- consolider les mesures institutionnelles préconisées et compléter les moyens financiers envisagés tout particulièrement à l'égard des pays tiers de la partie centrale et orientale du bassin danubien.

Tableau 1 : Principales initiatives européennes à moyen et à long terme relatives au système spatial et multimodal danubien.

\begin{tabular}{|l|l|l|l|}
\hline $\begin{array}{l}\text { Initiatives } \\
\text { communautaires }\end{array}$ & Champs d'interventions & $\begin{array}{l}\text { Horizon } \\
\text { temporel } \\
\text { visé }\end{array}$ & Échelles d'intervention \\
\hline
\end{tabular}




\begin{tabular}{|c|c|c|c|}
\hline $\begin{array}{lr}\text { 1) } & \text { Corridor } \\
\text { paneuropéen } & \text { du } \\
\text { Danube }^{10} & \text { (connu aussi } \\
\text { sous } & \text { l'intitulé } \\
\text { "Corridor } & \text { VII ») } \\
\text { officialisé en } 1994 . & \\
\text { (Figure 2a) } & \end{array}$ & $\begin{array}{l}\text { Initiative européenne } \\
\text { unique, à long terme, qui } \\
\text { porte directement sur le } \\
\text { bassin et le système } \\
\text { spatial danubien et seul } \\
\text { corridor paneuropéen } \\
\text { intégralement fluvial qui } \\
\text { constitue avec le canal } \\
\text { Rhin-Main-Danube, un } \\
\text { lien stratégique unique } \\
\text { entre la Mer Noire et la } \\
\text { Mer du Nord. }\end{array}$ & $\begin{array}{l}\text { Moyen } \\
\text { terme } \\
(2015)\end{array}$ & \begin{tabular}{|l} 
Ensemble des projets et des \\
mesures de développement \\
relatifs à l'axe du Danube ains \\
qu'à celui des infrastructures \\
multimodales connexes des pays \\
concernés régis par un protocole \\
d'accord formel et un secrétariat \\
(action politique avec très peu de \\
retombées directes concrètes \\
depuis 1994).
\end{tabular} \\
\hline $\begin{array}{l}\text { 2) Réseaux et projets } \\
\text { RTE-T d'intérêt } \\
\text { européen, approuvés } \\
\text { en } 2003 \text { (Figure } 2 b \text { ). }\end{array}$ & $\begin{array}{l}\text { Projets prioritaires } \\
\text { multimodaux du Réseau } \\
\text { transeuropéen } \\
\text { transport (RTE-T) des pays } \\
\text { de l'UE et des pays } \\
\text { candidats (uniquement) }\end{array}$ & $\begin{array}{l}\text { Long } \\
\text { terme } \\
(2020)\end{array}$ & $\begin{array}{l}\text { Une grande partie des réseaux } \\
\text { nationaux du bassin oriental du } \\
\text { Danube reste «de fait» non } \\
\text { couverte (particulièrement } \\
\text { Serbie, Croatie et Bosnie- } \\
\text { Herzégovine) ou peu concernée } \\
\text { (Roumanie et Bulgarie) }\end{array}$ \\
\hline 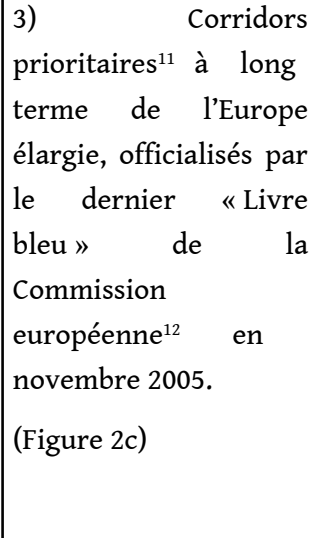 & $\begin{array}{l}\text { Corridors de transport } \\
\text { (multimodaux) projetés à } \\
\text { partir du RTE-T, à } \\
\text { destination des pays tiers } \\
\text { de l'Europe élargie }\end{array}$ & $\begin{array}{l}\text { Long } \\
\text { terme } \\
(2020)\end{array}$ & $\begin{array}{l}\text { Les axes prioritaires du réseau } \\
\text { européen composés par } \\
\text { l'extension de corridors } \\
\text { paneuropéens et de projets } \\
\text { prioritaires dans le cadre du RTE- } \\
\text { T, concernent un certain nombre } \\
\text { d'initiatives politiques (Groupes à } \\
\text { Haut Niveau I et II) et des } \\
\text { recherches européennes (PCRD) } \\
\text { dans le contexte des pays } \\
\text { membres et des pays tiers de la } \\
\text { région des Balkans et du Danube. }\end{array}$ \\
\hline
\end{tabular}

Figure 2

Figure 2a : Corridor paneuropéen VII (Carte officielle $\left.{ }^{13}, 2006\right)$

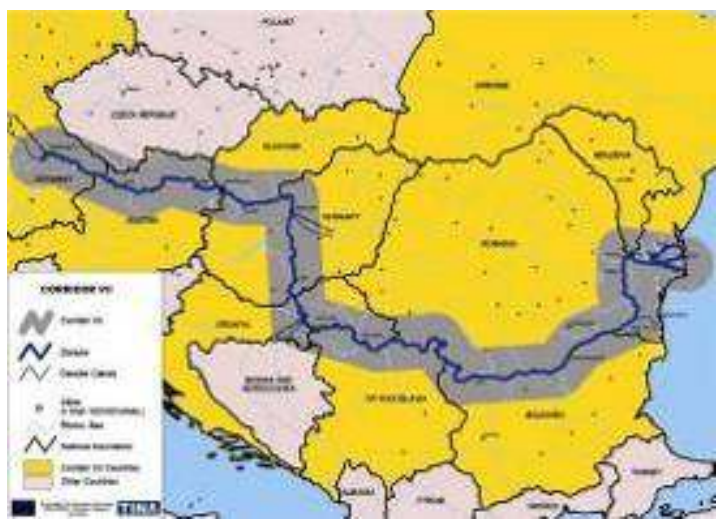


Figure 2b : Projets prioritaires RTE-T (Version adaptée issue de la carte officielle, 2003)

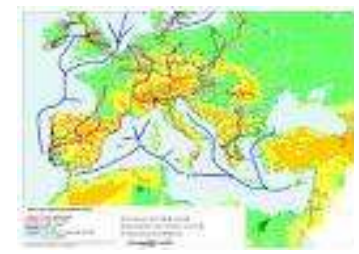

Figure 2c. Corridors prioritaires de l'Europe élargie (Carte officielle ${ }^{14}, 2005$ )

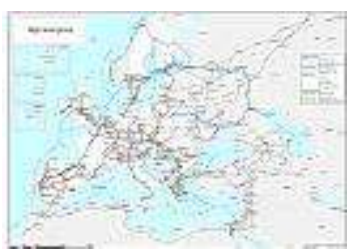

\section{Restructuration et différenciation des réseaux de transport}

6 La première étape de la démarche exploratoire vise à identifier et à présenter à partir de plusieurs illustrations modélisées, la différenciation spatiale des principaux réseaux de transport ${ }^{15}$ dans le bassin danubien, dans l'optique de pouvoir intégrer les changements recensés au début de la période transitoire (1990-1995) puis ceux qui se sont produits dans le contexte de l'élargissement en cours et notamment à la fin de la période d'observation (2000-2005). Les données publiques disponibles (fin 2005) et exploitables dans le contexte géographique étudié comme les données géo-référencées de la base officielle de la Commission européenne $\mathrm{GISCO}^{16}$, ainsi que certaines sources cartographiques nationales (données d'infrastructures et de trafic) ont permis de compiler progressivement les dimensions fonctionnelles et opérationnelles du Système d'information géographie du système spatial danubien, considéré dans un cadre global et européen (Figures $3 a$ et $3 b$ ). 
Figure 3a : Réseaux transeuropéens ferroviaires (réseau ferroviaire 1995-2004)

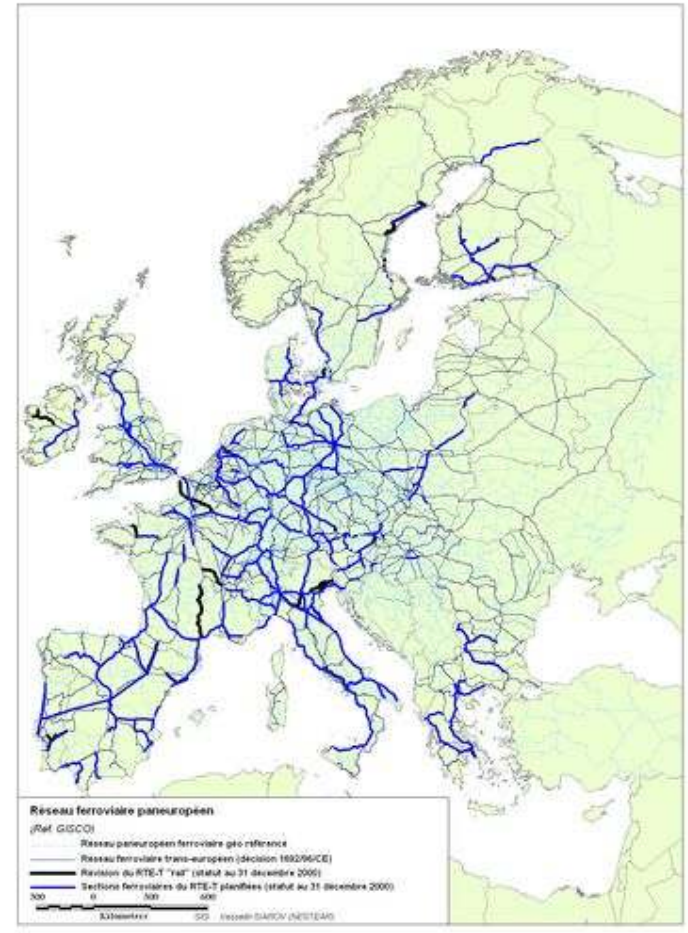

Figure 3b : Réseaux transeuropéens routiers (réseau routier 1995-2004)

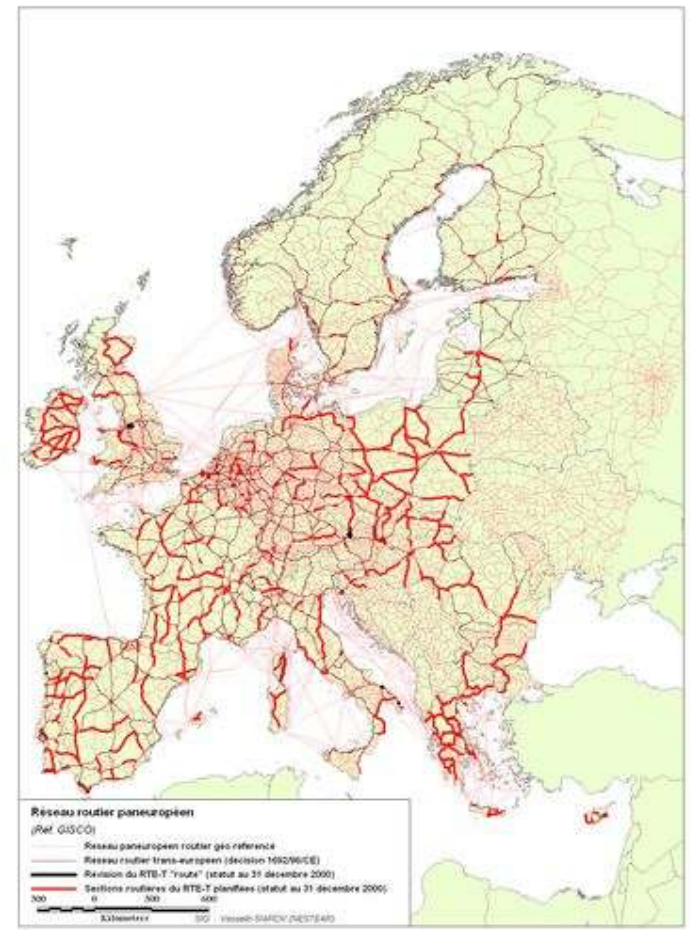

Source : (état des infrastructures, base 1995 et projets prioritaires en 2000, réf. GISCO v.4.0) 
Figure 4a : le système multimodal danubien articulé au transport ferroviaire

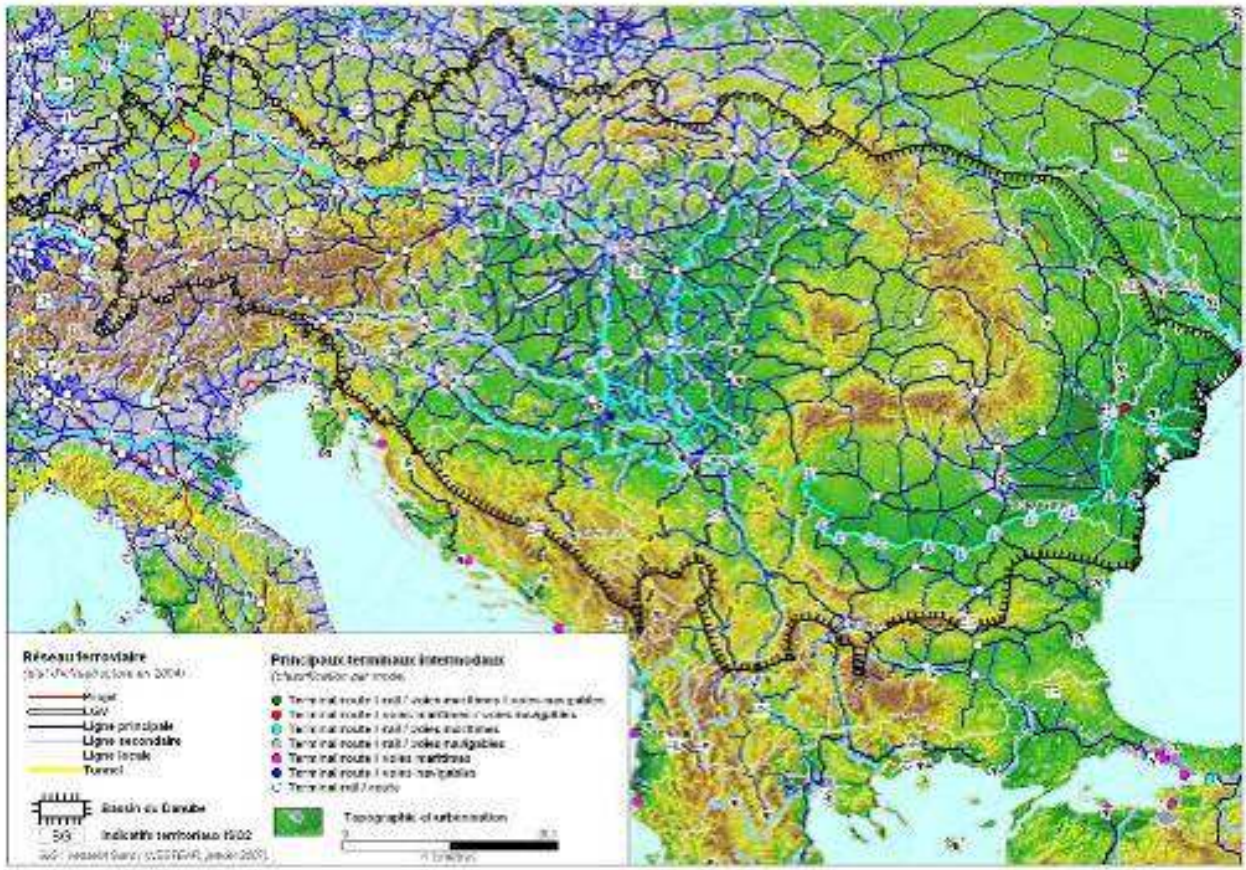

Figure $4 b$ : le système multimodal danubien articulé au transport routiera et $3 b$

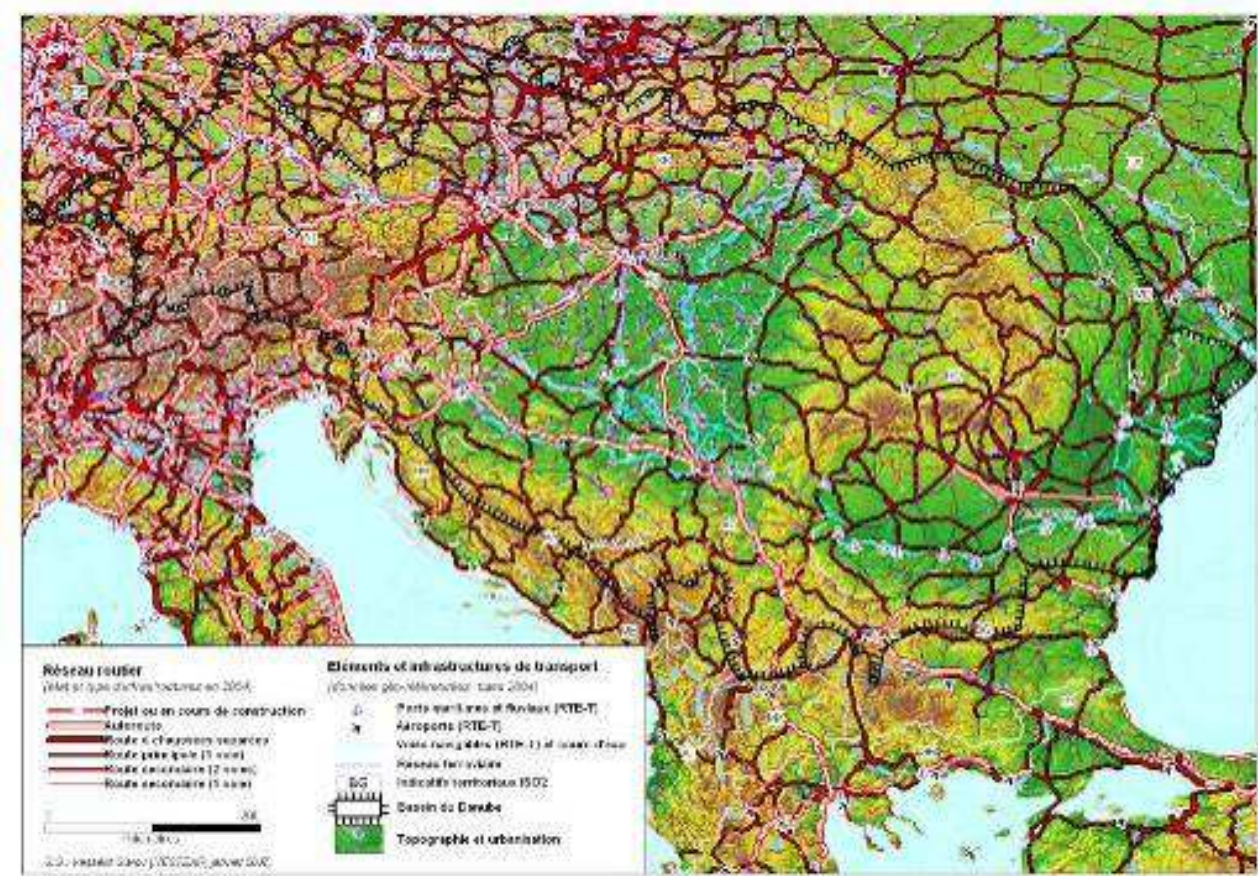

Source et méthodes : Réseaux et caractéristiques typologiques utilisés pour la mise au point du modèle de l'offre physique d'infrastructure 2000-2005 (i.e. répartition de la densité multimodale et spatiale)

7 Afin de pouvoir intégrer équitablement la répartition et l'évolution très hétérogènes des réseaux de transport (densité d'infrastructures routières, ferroviaires, voies navigables, etc.), puis celles des indicateurs socio-économiques (densités de 
population/richesses économiques) la base prototype du SIG ainsi constituée a pris en compte dans la première phase de ce travail, l'intensité moyenne kilométrique des infrastructures de transport (tous modes totalisés), à partir des unités cellulaires convenablement harmonisées (i.e. "raster» de 25 à $25 \mathrm{~km}$ ). Ainsi les données géoréférencées des infrastructures recensées au début de la période transitoire (i.e. 1990-1995) ont permis de composer la base initiale du SIG dédié, afin d'associer ultérieurement l'évolution temporelle correspondant à la fin de la période observée (2005) à partir d'un grand nombre de supports cartographiques et des observations personnelles relevées sur le terrain.

Figure 5a : Densité multimodale de transport en 1995

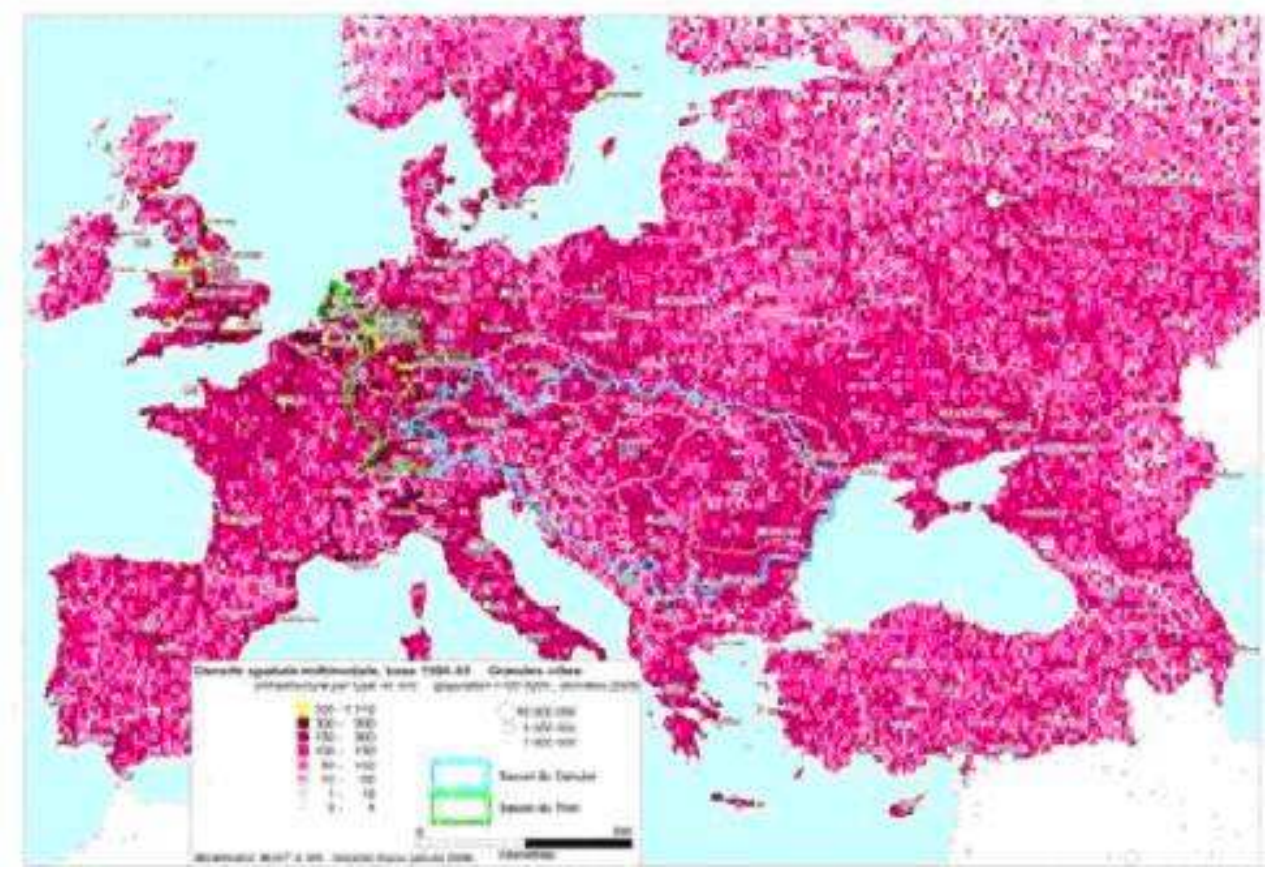

Figure 5b : Densité multimodale de transport en 1995 : liens de contiguïté ou de discontinuité multimodales

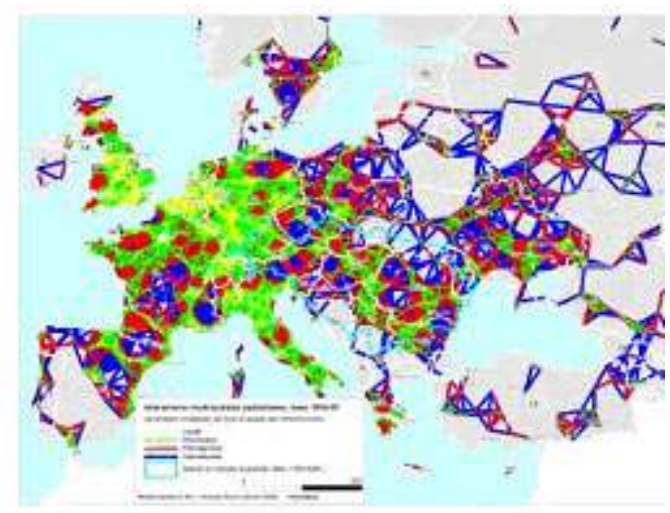

8 De façon globale mais aussi très détaillée, les projections et l'analyse des premières résultats illustrés (Figures $5 \mathrm{a}$ et $5 \mathrm{~b}$ ) ont confirmé et validé la position centrale et stratégique du système de transport danubien au sein de l'espace européen à partir notamment des densités moyennes multimodales totalisées ${ }^{17}$ par son bassin versant. 
Ces dernières bien qu'inférieures à celles du Rhin, s'avèrent être bien supérieures à la densité moyenne européenne, et ce sans compter la présence d'un très grand nombre d'interconnexions multimodales dans toute la partie occidentale du bassin (Allemagne, Autriche, Hongrie, Slovénie) qui constitue aussi un atout considérable pour le développement global régional de cette partie de l'Europe.

Cette centralité multimodale et géographique paraît encore plus visible à partir des extrapolations linéaires modélisées entre les aires de forte densité identifiée par le modèle, où l'axe fluvial « Rhin-Main-Danube $\aleph^{18}$, surtout dans la partie dorsale rhénane, apparaît comme une véritable colonne vertébrale du système multimodal danubien. Ainsi, le grand nombre d'aménagements dits de qualité (pôles logistiques, autoroutes, voies ferrées à plusieurs voies...) dans la partie nord-ouest du bassin (Figures 4a et $4 b)$ correspondsans surprise à des densités, voire à des contiguïtés plus importantes, par rapport aux extrémités périphériques.

Quant à la partie orientale, elle se caractérise par la présence d'une polarisation partielle et orientée vers l'est, résultant des infrastructures multimodales relativement denses et des liens bilatéraux a priori encore présents, dans la partie connexe à la Pologne, la Biélorussie et l'Ukraine. Ce constat est sans doute une conséquence des dispositifs des partenariats économiques hérités de l'époque du CAEM ${ }^{19}$.

Figure 6a : Densité multimodale de transport en 2005

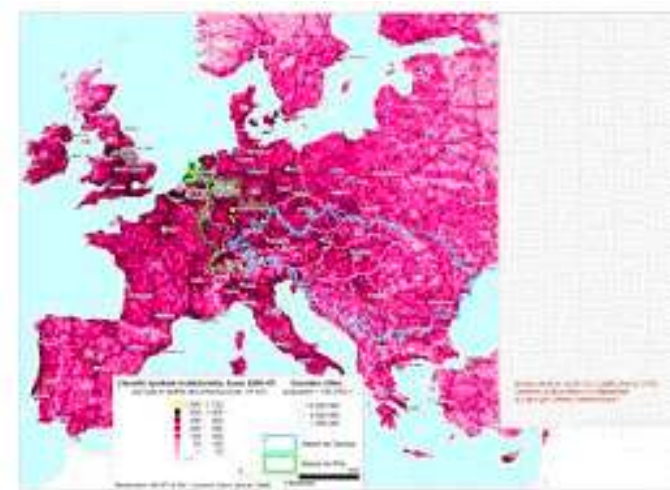

Méthode : densité kilométrique par unité spatiale, toutes infrastructures confondues avec distinction de type/capacité

Figure 6b : Densité multimodale de transport en 2005 : liens de contiguïté ou de discontinuité multimodales

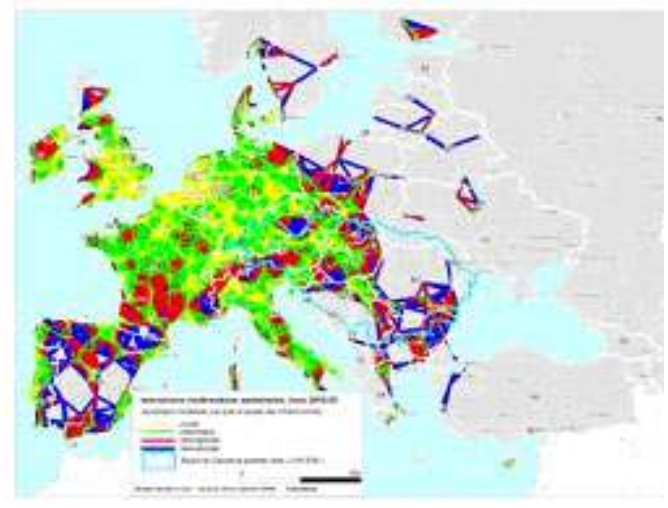


11 Après avoir recensé et intégré au sein du système composé du modèle, les aménagements d'infrastructure et de transport effectués jusqu'à présent (état des infrastructures période 2000-2005)y comprisles sections prioritaires ${ }^{20}$ du réseau transeuropéen de transport (RTE-T) et les maillons essentiels des réseaux nationaux, les différenciations entre parties occidentale et orientale du bassin (Figures 5 et 6)paraissent beaucoup plus concentrées et nettement plus faciles à identifier le long du corridor fluvial. En dehors de ce corridor principal, notamment dans les régions périphériques des Alpes Dinariques ou des Carpates, les carences (qualitatives et quantitatives) en infrastructures se sont révélées beaucoup plus profondes, ou du moins plus voyantes que dans le bassin occidental ou central.

En raison des conflits dans les Balkans, de la réorientation des échanges « Est-Ouest » et de l'élargissement inégalement amorcé en Europe centrale et orientale, certains maillons de transit, tels que les frontières et les points d'accès aux principaux ports et aéroports, se sont avérés souvent inadaptés à la croissance démesurée des besoins et des flux, tandis qu'une partie importante des voies navigables du Danube est restée longtemps isolée du trafic international (entre 1992 et 2005). En dépit de ce contexte géopolitique défavorable, la restructuration orientée et « occidentalisée » des densités et des liens multimodaux, constitue déjà une preuve ou une résultante d'un grand nombre d'infrastructures de qualité (aéroports, terminaux, autoroutes) aménagées récemment le long du bassin, en Hongrie, en Serbie, en Croatie, voire en Roumanie, sous les effets bénéfiques de l'intégration européenne et des échanges accélérés avec l'Union.

Dans ce sens, la persistance d'un fort contraste spatial entre l'axe central du Danube et ses périphéries extrêmes (nord-est et sud-ouest) correspondrait a priori à l'insuffisance d'infrastructures (Figure 6b), soit en raison du relief plus accidenté, soit en raison des échanges et de transports encore déficients entre l'UE et les territoires placés en périphérie du processus d'intégration européenne (Moldavie, Bosnie-Herzégovine, Albanie).Enfin, même s'il s'agit d'une interprétation macro-spatiale très partielle du système multimodal danubien, elle permet toutefois de montrer à partir des analyses spatio-temporelles effectuées l'émergence d'une certaine contiguïté linéaire et polycentrique, de plus en plus évidente, à partir des métropoles interconnectées, des régions communautaires frontalières, et surtout à proximité des points d'interconnexion maritimes/terrestres, notamment dans le nord de l'Adriatique et de la Mer Noire occidentale.

\section{Dynamique spatiale et temporelle des populations et des richesses}

14 Dans le double contexte de l'élargissement européen et de la mondialisation marquée notamment par des échanges croissants dans la région de la Méditerranée et de la Mer Noire, il apparaît utile voire indispensable de pouvoir estimer et de localiser, du moins globalement, l'intensité et la dynamique spatio-temporelle des mutations en cours. Les nouveaux outils expérimentés, ainsi que l'approche globale appliquée dans la première partie de cette recherche, se sont avérés particulièrement adaptés pour approfondir la démarche par une évaluation adéquate des mutations démographiques et économiques ${ }^{21}$ dominantes de la période récente (2000-2005), et d'un scenario tendanciel à l'horizon 2030 (Figures 7 et B). La suite de la démarche s'appuie sur 
l'intégration dans le modèle de la densité et de la croissance des populations et des richesses économiques, éléments primordiaux pour évaluer le potentiel et la dynamique du bassin danubien.

Figure 7a. Répartition de la population en 2005

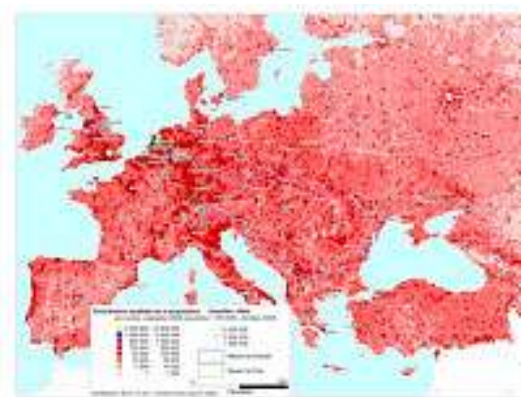

Figure 7b : Répartition de la population en 2005 : relations scalaires entre aires de forte concentration de population

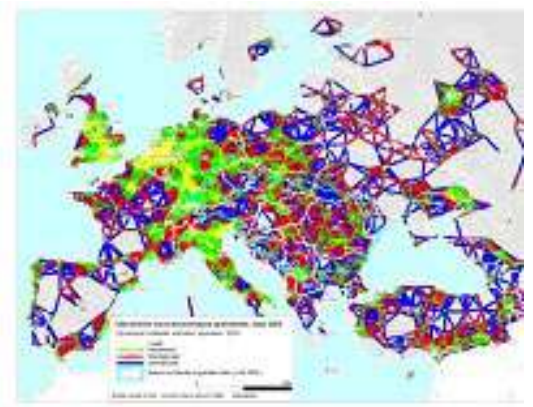

Figure 8a : Répartition du PIB en PPA en 2005 (en 1000 USD)

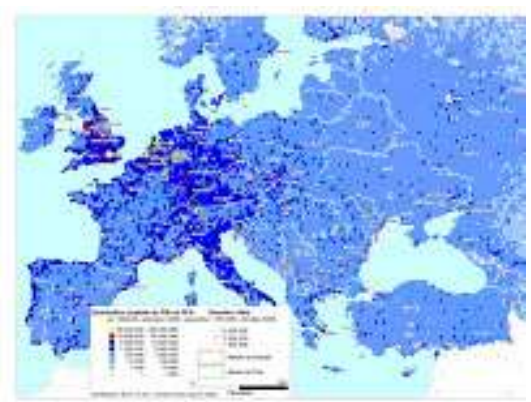


Figure 8b : Répartition du PIB en 2005 : relations scalaires entre aires de forte concentration de richesse

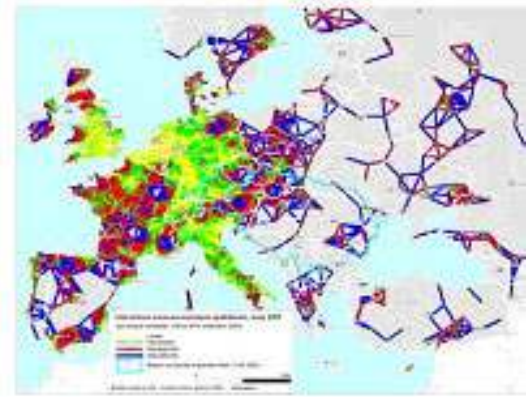

L'ensemble spatial danubien révèle des caractéristiques de concentration de villes et de populations très similaires à celles des régions voisines ${ }^{22}$ occidentales, orientales et méditerranéennes. Cependant, lorsque l'on analyse la répartition des densités démographiques et des richesses économiques du bassin, il apparaît que les relations dans sa partie sud et orientale présentent des discontinuités spatiales nettes et faciles à identifier, surtout au niveau des données économiques, même s'il s'agit d'une tendance a priori généralisée dans la périphérie est-européenne.

Il n'est pas surprenant de constater aussi que les densités les plus élevées en population et la concentration de richesses, observées dans le secteur de la dorsale européenne ainsi que pour les métropoles régionales (Salzbourg, Vienne, Bratislava, Budapest, Belgrade, Bucarest), soient étendues aux vastes plaines (Transdanubie et Valachie) disposant d'une contiguïté spatiale plus soutenue que les régions montagneuses (Alpes autrichiennes, Carpates et Balkans) avec le cœur européen.

De façon similaire, comme dans les analyses précédentes, on retrouve une forte polarisation (linéaire et/ou polycentrique) ordonnée par le gradient d'intégration européenne : plus les localités sont éloignées du cœur économique occidental et des frontières communautaires, plus le développement global économique parait insuffisant, à l'exception de quelques liens émergents sur la façade maritime de la Mer Noire.

Pour davantage mettre en valeur les aspects prévisionnels des analyses thématiques, il convient d'introduire au sein de la même base opérationnelle un module supplémentaire de projections temporelles (référence 2005, projections 2030), afin d'illustrer l'évolution possible de l'espace danubien et de mieux appréhender trois ordres de mutations en cours :

- l'ouverture et la stabilisation des économies en transition (partie centrale et orientale du bassin de 1990 à nos jours) ;

- l'intégration européenne (Autriche en 1995 et République Tchèque, Slovaquie, Hongrie, Slovénie en 2004, Bulgarie et Roumanie en 2007);

- la libéralisation des marchés (services, transports et finances), la mondialisation des échanges (par le biais de la périphérie maritime : Atlantique, Adriatique, Mer Noire) et l'extension, voire la consolidation des nouveaux corridors de transport.

Dans ce cadre géopolitique très dynamique, en l'absence de scénarios formels de croissance démographique ou économique, applicables à long terme et à l'échelle des pays et des régions concernés, il serait intéressant d'associer, à titre expérimental, les tendances et les croissances récentes (accroissement naturel démographique et taux de croissance économique ${ }^{23}$ ) au support opérationnel et fonctionnel déjà constitué,dans 
l'optique de projeter et de prévoir les perspectives de développement global, danubien et européen d'ici 2030.

Figure 7c : Projection tendancielle de la population en 2030

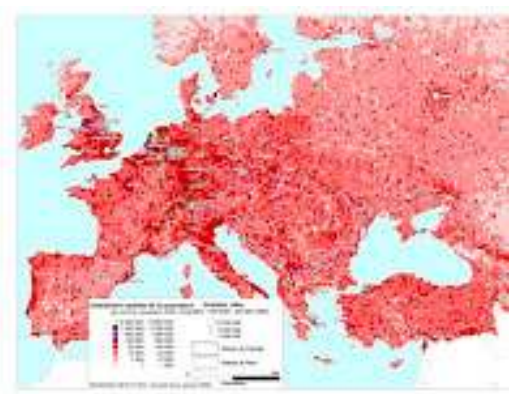

Figure 7d : Projection de la population en 2030 : relations scalaires entre aires de forte concentration de population

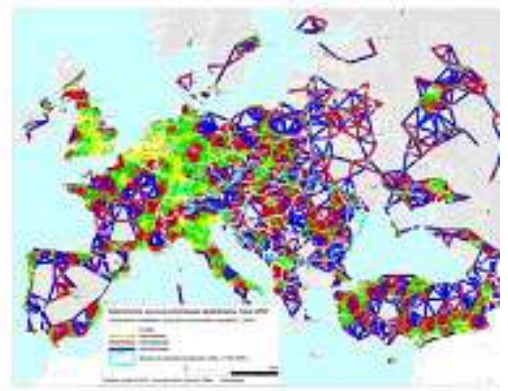

Figure 8c : Projection tendancielle du PIB en 2030 (en 1000 USD

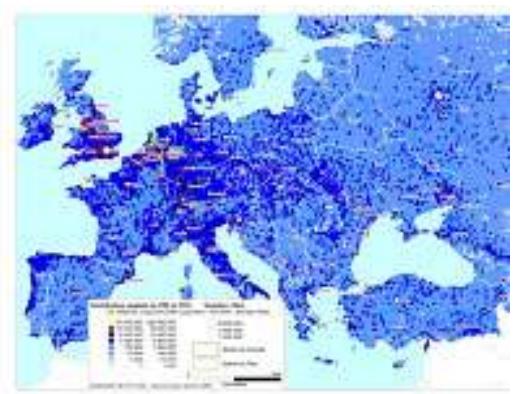

Figure 8d: Projection du PIB en 2030 : relations scalaires entre aires de forte concentration de richesse

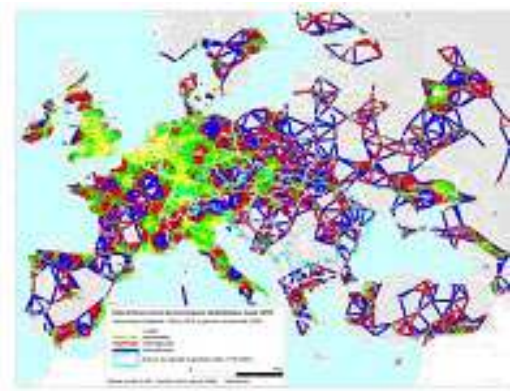


20 Conformément aux projections analysées pour 2005 et au scénario de références appliquées,l'intensité des polarisations économiques projetées à long terme (horizon 2030)devient en effet beaucoup plus dense, mieux agencée et plus équilibrée entre les parties orientale et occidentale du basin danubien (Figures $7 \mathrm{c}$ et $7 \mathrm{~d}$ et $8 \mathrm{c}$ et $8 \mathrm{~d}$ ).Ainsi, la concentration supérieure et l'apparition d'un très grand nombre de nouveaux liens polycentriques à l'échelle du bassin laissent espérer qu'il est toutefois possible d'estomper à terme, du moins partiellement, les oppositions spatiales les plus contrastées entre ses les deux parties du bassin.

21 A peine révélée à la fin de la période d'observation (2000-2005), la dynamique interrégionale projetée à l'horizon 2030 s'avère a priori stimulée par les nouveaux pays membres d'Europe centrale, où il est possible de distinguer très nettement (en 2030 plus qu'en 2005) l'émergence d'un nouveau pivot économique européen ${ }^{24}$.La traduction de ces prévisions en échanges économiques et par extension, en flux de transport (tous modes et échelles confondus), indique une croissance globale d'ici 2030, susceptible de générer une augmentation totale du trafic multimodal dans l'espace danubien de l'ordre de 2 à 2,5 fois supérieure au niveau actuel (base 100, fin 2005).

22 La seule incertitude presque inquiétante concernerait les Balkans et particulièrement l'espace compris entre la Bosnie-Herzégovine, la Serbie et la Bulgarie (Figures 7 et 8), où les contraintes politiques et économiques cumulées depuis une dizaine d'années ainsi que les statistiques déficientes, tendances rompues et données souvent absentes, sont $a$ priori à l'origine de la fracture spatiale persistante entre la partie occidentale et orientale des Balkans, même à l'horizon 2030. La confirmation de ces tendances alarmantes, validées également par les estimations modélisées pour 2005 ainsi que par les prévisions de 2030, risquent d'accroître davantage les disparités régionales, en raison notamment du dépeuplement des campagnes et de la persistance du taux de croissance démographique négatif ${ }^{25}$ dans une grande partie des régions du bassin oriental (Bulgarie, Hongrie, Moldavie et Ukraine).

23 Dans ce contexte, les espaces économiques identifiés comme moins bien développés (surtout en 2005 et partiellement en 2030), vont sans doute parvenir à raccorder, à terme, le système central danubien grâce à un nombre limité de liens linéaires au départ du pourtour maritime bulgare, roumain ou adriatique et en direction des plaines d'Europe centrale (Hongrie, Croatie, Slovaquie). Le reste des Balkans, notamment la Serbie, la Bosnie, le sud de la Transylvanie et une grande partie de la Bulgarie, a priori sous le coup des conflits récents (1991-2000) mais aussi de l'intégration européenne différée, sont toujours, de fait, isolés de la partie la plus dynamique du système. En effet, en raison du contexte économique défavorable (guerres et conflits, instabilité politique et transition difficile dans la partie sud et orientale du bassin), ces quinze dernières années, seules les parties occidentales et centrales danubiennes sont pour l'instant, et a priori pour les années à venir, les seules régions en mesure de bénéficier pleinement de l'intégration et de la continuité spatiale avec le reste de l'Union.

24 Toutefois, compte tenu du contexte évolutif et du fait qu'il s'agit ici de modélisations, les incertitudes de développement sont telles qu'il n'est pas exclu aussi de voir prochainement un développement de toute la région des Balkans, tiré par l'intégration européenne et amplifié par la croissance économique plus élevée que celle de la moyenne continentale européenne ${ }^{26}$.

25 Enfin, il convient, de souligner, ici, le caractère strictement opérationnel et expérimental des scénarios appliqués dans une région vaste et contrastée comme le 
bassin danubien. A ce titre, les résultats présentés, bien que préservés de toute influence volontariste, personnelle et politique, méritent sans doute d'être ajustés ou mieux précisés (finesse des données et découpage territorial ${ }^{27}$ ). Néanmoins, ces derniers mettent en évidence trois phénomènes spatiaux :

- La répartition globale des infrastructures multimodales, orientées et structurées a priori par l'axe du corridor fluvial danubien (état en 1995 et 2005);

- Le chevauchement dense et continu de la demande potentielle dans une grande partie du bassin occidental, contrastant avec les dynamiques de la partie orientale, même à l'horizon 2030 ;

- La puissance des outils expérimentés (modèle spatial et de SIG), lorsqu'ils sont utilisés pour identifier, définir et projeter avec une précision pratiquement « kilométrique » les enjeux stratégiques, socio-économiques et logistiques du système danubien.

\section{Convergence, cohésion et perspectives de développement global}

La recherche de cohésion territoriale et de convergence régionale, aux niveaux de l'Union et des pays candidats à l'adhésion, souvent évoquée par de nombreux décideurs (politiques et économiques) et préconisée par un grand nombre de directives européennes, fait partie des problématiques les plus étudiées d'aménagement des territoires, de planification des transports et du développement durable européen. de références spatiales ainsi que les dernières avancées informatiques ont rendu possible l'utilisation simultanée, à des fins prévisionnelles, comparatives ou illustratives, d'un grand nombre d'informations pertinentes, permettant d'analyser et surtout de mieux appréhender les mutations permanentes des phénomènes spatiotemporels. C'est ainsi que les questions de repérage des dynamiques territoriales existantes ou potentielles - ont motivé la recherche de solutions innovantes et plus pragmatiques grâce à l'intégration virtuelle de données spatialisées au sein de systèmes d'information géographique, adaptés aux contextes étudiés et capables de comparer un très grand nombre de résultats modélisés dans une démarche temporelle ou multiscalaire.

A titre d'essai, la première tentative de faire converger la dynamique spatio-temporelle des infrastructures, des populations et des richesses économiques modélisées, dans l'optique de simuler, par le biais du modèle, un équilibre ${ }^{28}$ opérationnel de développement global et de cohésion territoriale optimale n'a pas donné de résultats probants pour quasiment toute la partie orientale du système danubien. Ce résultat exploratoire constitue en soit un signe révélateur des fortes disparités démographiques, économiques et surtout des énormes besoins en infrastructures de transport conformes aux exigences internationales de qualité, de capacités et de protections environnementales (autoroutes, lignes ferroviaires électrifiées et à plusieurs voies, ports, aéroports et terminaux). Dans l'optique de consolider, voire de cerner partiellement le potentiel de cohésion même minimal des relations non convergées par la première investigation, une seconde simulation, calibrée ${ }^{29}$ sur le bassin danubien, et ajustée par le poids gravitaire du système de transport, a été conceptualisée. 
simulation finale a permis de révéler la convergence des éléments constitutifs du système central et occidental du bassin, à la différence de la partie orientale, toujours très fragmentée, où l'émergence d'un nouvel axe bipolaire, compris entre le port bulgare de Varna et la capitale roumaine, Bucarest, constituerait a priori un début de coopération et de développement interrégional très pertinent dans le contexte du dernier élargissement européen (Figure 9).

Figure 9 : Modélisation de la cohésion du bassin danubien définie par l'offre d'infrastructures

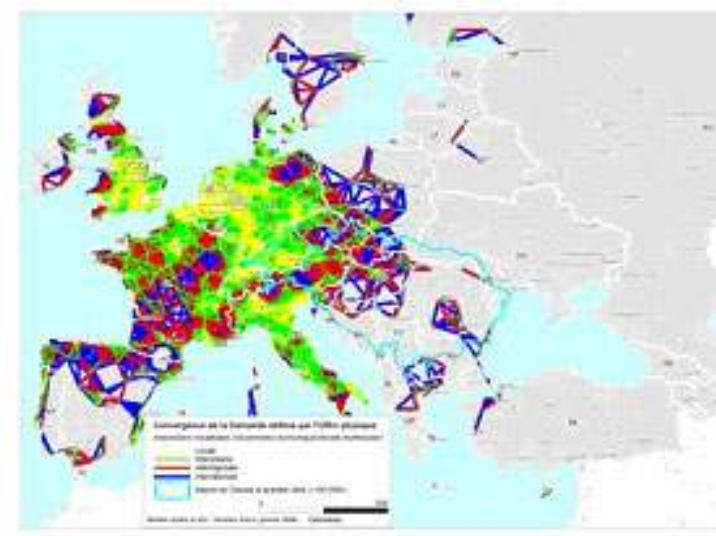

Sources et méthodes : Données totalisées des infrastructures, des populations et des PIB rastérisées, base 2005Relations de convergence linéaires modélisées à partir des interactions (virtuelles simulées) entre les aires de fortes densités d'infrastructures, de populations et de richesses économiques.

Cette ultime illustration des différenciations spatiales danubiennes fournit de façon encore plus explicite plusieurs réponses à la problématique posée. La première souligne l'importance cruciale de l'intégration des infrastructures de transport lors des conceptions modélisées à vocation d'analyse, de prospective et de prévision territoriales, en raison de leur apport structurant au développement global et interrégional, surtout dans le cadre des programmes européens à long terme visant à maitriser voire à mieux orienter les polarisations spatiales et les mutations en cours (par le biais d'aides et de fonds spécifiques). La seconde réponse, et la plus importante dans le contexte concret de ce travail, établit un état des lieux, bien que général, mais pertinent et argumenté, qui confirme le bon potentiel de convergence du bassin occidental à proximité des pôles de forte polarisation économique et seulement aux points d'accès stratégiques mer/terre dans sa partie orientale. Enfin, l'absence quasiintégrale de cohésion spatiale dans les Balkans pourrait être au moins partiellement compensée par la formation progressive de nouveaux avant-postes ${ }^{30}$ stratégiques, situés aux confins de la périphérie euro-danubienne, et à la "porte d'entrée " des futures extensions eurasiennes (Mer Noire, voies navigables russes à très grand gabarit, bassin caspien, etc.).

\section{Conclusion}

La série d'illustrations ainsi présentées a confirmé la convergence partielle interrégionale et l'absence de cohésion territoriale intégrale au sein du système danubien étudié. Conceptualisée et appliquée dans le contexte spécifique à la fois danubien mais aussi européen, la modélisation expérimentée a permis de positionner 
un certain nombre de faits ou d'évolutions plus ou moins supputés, mais difficiles à identifier autrement. Plusieurs éléments doivent être soulignés.

En l'état actuel supposé des caractéristiques intégrées, le système spatial danubien confirme pleinement sa position centrale au sein de l'espace européen, un fait crucial qui ne lui permet pas pour autant, du moins pour l'instant, d'assurer une cohésion spatiale. Il apparaît que la moitié occidentale du bassin, structurée par la proximité de l'axe rhénan et par les bienfaits des échanges intracommunautaires, représente le moteur et les aiguilles gravitaires de ce nouveau système économique et logistique d'importance européenne. La seule objection au développement intégral de cet espace stratégique pour l'Union concernerait a priori la partie sud et orientale du bassin, où une partie importante apparait sans surprise, encore absente, voire complètement déconnectée du système spatial et multimodal danubien. Enfin, à condition que les composantes du système opérationnel soient modélisées selon l'offre d'infrastructure multimodale conformément aux demandes institutionnelles de développement régional,il est possible d'améliorer sensiblement et à terme la cohésion territoriale et la convergence interrégionale de l'espace danubien.

Bien que limité et encore très peu exploité, le potentiel socio-économique et logistique du système danubien est immense. En dépit de la déficience chronique en ressources et en infrastructures, particulièrement visibles dans la partie inférieure du bassin, nous assistons déjà à l'évolution la plus importante de toute l'histoire récente de cette partie de l'Europe, traduite notamment, par l'élargissement européen, l'essor maritime et fluvial $^{31}$ et le développement global interrégional, déjà engagés. Dans ce contexte, l'ajustement équitable des politiques de cohésion et de convergence européennes, tout comme la prospection possible de nouvelles solutions logistiques, mieux adaptées aux dispositifs d'infrastructure, constituent un enjeu important ainsi qu'une garantie au développement danubien, au moment où les pays occidentaux cherchent à établir de nouveaux corridors stratégiques vers la Russie, le bassin Caspien, l'Asie centrale, la Turquie, voire le Proche-Orient.

\section{BIBLIOGRAPHIE}

Bavoux (Jean-Jacques), Charrier (Jean-Bernard), Transport et structuration de l'espace dans l'Union européenne, Paris : MASSON, 1994.

Commission Européenne, Des réseaux pour la paix et le développement, Extension des grands axes transeuropéens de transport vers les pays et régions voisins, Rapport de Groupe à Haut Niveau (HLG II), Bruxelles, novembre 2005.

Merlin (Pierre), Géographie, économie et planification des transports, Paris : PUF, 1991.

Navigation Ports \& Industries, Une activité disparate entre Est et Ouest, avril 2005, pp. 180-192.

NEA and alii, Scenarios, Traffic Forecasts and Analysis of Corridors on the Trans-European Transport Network, TEN-STAC Final Report - D8, Brussels: European Commission, 2004. 
Reynaud (Christian), Siarov (Vesselin) et alii, Corridors eurasiatiques et les enjeux des liaisons de Fret entre l'Europe et l'Asie, Rapport scientifique, Gentilly : NESTEAR, 2006.

Siarov (Vesselin), Evaluation stratégique des projets d'infrastructure de transport : Le corridor paneuropéen $N^{\circ} 10$ "Salzbourg-Thessalonique ", Thèse de doctorat en aménagement et urbanisme, Saint-Denis : Institut d'études européennes - Université Paris 8, 2003.

Siarov (Vesselin), Intégration des échanges et des transports à l'échelle de l'Europe élargie: Analyse spatiale intermodale dans la région de la Méditerranée et de la Mer Noire, Paris : Colloque de clôture Action COST 340, 2005.

Siarov (Vesselin), Integration of Central and Eastern European countries in the Pan-European Transport System, Report on transport in the Balkan Region, Paris: European Conference of Ministers of Transport - Committee of Deputies CEMT/CS (2000)4, 2000.

Siarov (Vesselin), La nodalité à l'échelle paneuropéenne, Université de Cergy-Pontoise : Journées de géographie des transports, 2004. [En ligne] http://www.nestear.net/home_fr/index_fr.html

Siarov (Vesselin), Pertinence et potentiel de l'intermodalité dans la région de la Méditerranée et de la Mer Noire, Kiev : Séminaire CEMT/CEE-ONU Intermodalité Europe - Asie pertinence et potentiel, 2004. [En ligne] http://www.nestear.net/home_fr/Docs/INTERMODALITE_MED.PDF.pdf

***, Qu'en est-il de la voie d'eau pour le fret demain en France et en Europe, Rapport d'information 366 (2001-2002), Paris : Sénat - Commission des affaires économiques, 2002.

TINA Secretariat, Identification of the network components for a future Trans-European Transport Network in Bulgaria, Cyprus, Czech Republic, Estonia, Hungary, Latvia, Lithuania, Poland, Romania, Slovakia and Slovenia, TINAFinal Report, Vienna : TINA Groups, 1999.

\section{Sites Internet :}

\begin{tabular}{|l|l|}
\hline SITE INTERNET & SOURCE \\
\hline $\begin{array}{l}\text { http://www.cia.gov/cia/publications/ } \\
\text { factbook/ }\end{array}$ & CIA - The World Factbook \\
\hline $\begin{array}{l}\text { http://devdata.worldbank.org/query/ } \\
\text { default.htm }\end{array}$ & The World Bank Group - World Development Indicators \\
\hline $\begin{array}{l}\text { http://www.libraries.psu.edu/maps/ } \\
\text { onlinedata.htm }\end{array}$ & $\begin{array}{l}\text { The Pennsylvania State University Libraries - Digital Chart } \\
\text { of the World }\end{array}$ \\
\hline http://www.gazetteer.de/ & World Gazeetteer \\
\hline http://earth-info.nga.mil/gns/html/ & GEOnet Names Server - GNS \\
\hline http://fr.wikipedia.org/ & L'encyclopédie libre WikipédiA \\
\hline http://www.tinavienna.at/corridor7/index.php & The Danube Corridor (Corridor VII) \\
\hline $\begin{array}{l}\text { http://europa.eu.int/comm/ten/transport/ } \\
\text { external_dimension/ } \\
\text { doc/2005_12_07_ten_t_final_report_fr.pdf }\end{array}$ & $\begin{array}{l}\text { des réseaux pour la paix et le développement : Extension } \\
\text { et régions voisins }\end{array}$ \\
\hline
\end{tabular}




\begin{tabular}{|l|l|}
\hline $\begin{array}{l}\text { http://ec.europa.eu/enlargement/glossary/ } \\
\text { terms/ipa_fr.htm }\end{array}$ & $\begin{array}{l}\text { EUROPA, Elargissement, Instrument d'aide de préadhésion } \\
\text { (IPA) }\end{array}$ \\
\hline http://www.globeco.fr/ & GLOBECO \\
\hline http://www.ums-riate.com ). & $\begin{array}{l}\text { Réseau Interdisciplinaire pour l'Aménagement du Territoire } \\
\text { Européen (RIATE) } \\
\text { Unité Mixte de Service 2414 }\end{array}$ \\
\hline
\end{tabular}

\section{ANNEXES}

\section{Encadré méthodologique}

Définitions du modèle de contiguïté territoriale et du SIG

La modélisation de la contiguïté représente une nouvelle approche spatio-temporelle d'analyse et de prospective territoriale, assistée par des systèmes d'information géographique (SIG). C'est également un nouvel outil d'aide à la décision qui vise à capitaliser le dispositif local et global, traduit par l'inscription spatiale des informations vectorielles géo-référencées (réseaux de transport, population et richesses économiques, etc.) sur une base parfaitement homogène (raster cellulaire) dans l'optique d'assurer le suivi permanent ou ponctuel des dynamiques spatiales et de prévoir le développement global des systèmes complexes et intégrés. Issue d'un grand nombre d'expérimentations fonctionnelles et opérationnelles multi-échelles (Monde, Europe, Méditerranée, Turquie, Chine), dans sa version finale, cette nouvelle approche de prospective spatio-temporelle comporte deux modules essentiels permettant de modéliser aisément les interactions complexes et d'identifier avec une précision kilométrique l'évolution polycentrique des territoires considérés.

Démarche modélisatrice

La démarche opérationnelle multi-scalaire et la classification hiérarchique opérationnelle et/ou fonctionnelle des graphes modélisés constituent à la fois une démonstration de contigüité territoriale ainsi qu'une base de référence pour des affectations directes ou indirectes.

Le module d'interactions spatio-temporelles d'infrastructures multimodales (MIST-IT) vise à intégrer la densité des infrastructures de transport totalisées par unité de surface " raster » afin de composer l'offre physique d'infrastructure, alors que le module d'interactions spatio-temporelles socio-économiques (MIST-SE) permet de situer la distribution spatiale et tendancielle de la demande globale (illustrée ici par la densité de la population ou des richesses économiques i.e. produit intérieur brut en parité de pouvoir d'achat).

Inaugurée en décembre 2005, par l'auteur de ce document, cette nouvelle conceptualisation d'analyse spatiale a permis de valider l'applicabilité du modèle de contiguïté territoriale afin de confirmer, à partir des nombreuses expérimentations testées, ces performances opérationnelles : 
Outil puissant de prospective territoriale et instrument efficace d'aide à la décision (pédagogique, opérationnel et fonctionnel), capable d'intégrer la localisation fine et l'évolution globale d'un grand nombre de références (données rasters ou vectorielles) mais aussi de simuler des interactions linéaires et polycentriques au sein des systèmes complexes (territoires/réseaux/produits/acteurs...) ;

Outil additionnel aux approches traditionnelles d'analyse spatiale et de prospective territoriale susceptible d'assister la mise en place de réseaux (choix de projets d'infrastructure, de liens prioritaires, etc.) ou de graphes orientés au sein des modèles de prospective territoriale, de navigation ou simplement de projection directe/ indirecte à partir notamment des affectations classiques et économétriques (ex. modèles gravitaires) ou par le biais de modélisations de nouveaux types (polycentriques, inter-polaires, multi-scalaires...)

Système d'information géographique

Les SIG permettent de gérer des données alphanumériques spatialement localisées, ainsi que des données graphiques permettant d'afficher ou d'imprimer plans et cartes. Ses usages couvrent les activités géomatiques de traitement et de diffusion de l'information géographique.

Le rôle du système d'information est de proposer une représentation plus ou moins réaliste de l'environnement spatial en se basant sur des graphiques primitifs tels que des points, des vecteurs (arcs), des polygones ou des maillages (raster). À ces graphiques sont associées des informations qualitatives (route, voie ferrée, forêt, etc.) quantitatives ou toute autre information contextuelle.

Les données géographiques (du SIG) possèdent quatre composantes :

les données géométriques renvoient à la forme et à la localisation des objets ou phénomènes ;

les données descriptives (qui font partie des données attributaires) renvoient à l'ensemble des attributs descriptifs des objets et phénomènes à l'exception de la forme et de la localisation ;

les données graphiques renvoient aux paramètres d'affichage des objets (type de trait, couleur...);

les métadonnées associées, c'est à dire les données sur les données (date d'acquisition, nom du propriétaire, méthodes d'acquisition...).

Source : sélection et définition effectuées par l'auteur à partir de plusieurs sources, dont http://fr.wikipedia.org/

\section{NOTES}

1. Les notions d'« espace européen » ou d'« espace européen élargi » considérées dans cet article font référence à l'espace européen continental. Ce dernier est différent de celui des pays membres de l'Union, dénommé également « espace communautaire ».

2. La notion opérationnelle de "système spatial danubien " utilisée dans ce document recouvre le bassin du Danube (défini par les limites naturelles du bassin versant) dans son articulation 
avec les dimensions spatiales intégrées par le modèle (territoires, infrastructures de transport, populations et richesses nationales et/ou régionales produites).

3. La Croatie, à la différence des autres pays tiers, est dotée depuis fin 2005 d'un statut officiel de pays candidat à l'Union et à ce titre est éligible aux programmes spécifiques d'aide communautaire, attribués pour faciliter la préadhésion.

4. Nomenclature des unités territoriales statistiques utilisée par Eurostat (NUTS).

5. Les outils opérationnels (SIG et modèle spatial) ainsi que l'approche multi-scalaire utilisée sont en mesure d'aborder des analyses encore plus fines et détaillées, susceptibles d'extrapoler la multiplicité des impacts de différenciation ou d'intégration à des échelles variables (locale, interurbaine, interrégionale et internationale).

6. Modèle de contiguïté territoriale, assisté d'un SIG, conceptualisé par l'auteur (déc. 2005) pour intégrer, analyser et projeter la dynamique spatio-temporelle de l'espace territorial découpé, recomposé et modélisé dans un contexte à la fois danubien et européen (voir l'encadré méthodologique).

7. Dans le contexte considéré ici les richesses économiques sont résumées par le produit intérieur brut en parité du pouvoir d'achat (PIB en PPA), qui constitue le seul indicateur international permettant de comparer équitablement l'importance des valeurs ajoutées brutes ainsi que les potentiels économiques nationaux. (cf. $h t t p: / / w w w . g l o b e c o . f r /)$

8. Les projets prioritaires à court terme destinés aux pays membres (dont les nouveaux accédants: Slovénie, Hongrie, Slovaquie, etc.) sont régis par les fonds spécifiques communautaires (budget RTE-T, fonds structurels et de cohésion, alors que ceux alloués aux pays candidats (Bulgarie et Roumanie avant 2007, et Croatie) sont couverts presque intégralement par les programmes PHARE et ISPA (IPA - Instrument de Préadhésion, depuis le $1^{\mathrm{er}}$ janvier 2007). Les pays tiers des Balkans occidentaux, n'étant pas éligibles (du moins jusqu'à la signature d'un accord formel de préadhésion) ne sont actuellement concernés que par les fonds de soutien spécifique CARDS et OBNOVA. L'instrument d'aide de préadhésion (IPA) est l'aide de préadhésion unique pour la période 2007-2013 qui apporte une assistance financière aux pays candidats et aux pays candidats potentiels. À ce titre, il remplacera les programmes Phare, ISPA et SAPARD, l'instrument de préadhésion spécifique pour la Turquie ainsi que le programme CARDS (cf. http://ec.europa.eu/enlargement/glossary/terms/ipa_fr.htm).

9. Les travaux de recherche produits dans le cadre de l'Observatoire en Réseau de l'Aménagement du Territoire Européen (Orate - Espon), intégrant depuis peu (fin 2004) des analyses spatiales et thématiques en matière d'aménagement européen, y compris sur certains pays danubiens (dont Bulgarie et Roumanie, depuis 2006), n'ont pas pu être pris en compte dans le modèle en raison de la publication très récente des rapports de recherche. (cf. http:// www.ums-riate.com).

10. Corridor paneuropéen, déclaré prioritaire lors de la seconde conférence paneuropéenne sur les transports (Crète, 1994). Le corridor s'étale sur $2415 \mathrm{~km}$, il comporte 44 ports fluviaux et maritimes d'une distance moyenne de $55 \mathrm{~km}$ et dispose entre autre de connexions multimodales stratégiques avec 4 des 10 Corridors paneuropéens (Corridors IV, V, IX et X). Depuis 1994, dix pays sont directement concernés par le corridor fluvial du Danube et ont déjà signé le protocole d'accord pour sa promotion et son développement (Allemagne, Autriche, Slovaquie, Hongrie, Croatie, République Fédérale de Yougoslavie i e. Serbie actuelle, Roumanie, Bulgarie, Moldavie et Ukraine).

11. Une dizaine de corridors multimodaux terrestres ainsi que plusieurs autoroutes de la mer ont été définis comme prioritaires et d'intérêt stratégique européen en décembre 2005.

12. Cf. Commission européenne, « Des réseaux pour la paix et le développement, Extension des grands axes transeuropéens de transport vers les pays et régions voisins ", Rapport de Groupe à Haut Niveau II, Bruxelles, 2005.

13. Cf.: http://www.tinavienna.at/corridor7/index.php 
14. Cf. : $\quad$ http://europa.eu.int/comm/ten/transport/external_dimension/doc/ 2005_12_07_ten_t_final_report_fr.pdf

15. Les infrastructures de transport (i.e. offre physique multimodale d'infrastructure au sein du modèle) sont transposées ici par les références spatiales des réseaux de transport (rail, route, voies navigables, aéroports, ports et terminaux multimodaux), agrégés et intégrés par le modèle spatial.

16. Le référencement spatial du bassin danubien repose sur les fichiers thématiques (SIG) de la base GISCO («DATABASE GISCO», v.4.0, 2003).

17. A titre d'exemple, en 1995 , le système multimodal danubien comptait approximativement $9 \%$ du total kilométrique européen contre seulement 7,5\% de la superficie totale continentale. En 2005, les proportions estimées (par le modèle) sont beaucoup plus importantes (supérieures à 13\%). 18. En dépit de l'inauguration du canal Rhin Main Danube en 1992, le plus dense système fluvial à gabarit européen (supérieur à 1350 t soit égal ou supérieur à la 'classe IV') n'a pas pu réaliser, en raison des conflits yougoslaves, les effets positifs escomptés.

19. Conseil d'assistance économique mutuelle de l'ancien bloc de l'Est. Sa dissolution en juin 1991 a marqué la rupture de fait des relations économiques et commerciales entre les anciens pays partenaires.

20. Compte tenu du faible nombre de réalisations prioritaires relevant des dispositifs du RTE-T, à cette étape de la démarche, seules les caractéristiques spatiales et typologiques des ports et des aéroports ont été intégrées dans le système multimodal du SIG (état 2000-2005).

21. La base spatiale SIG des populations comporte les dernières données disponibles (fin 2005) de la population urbaine mondiale répartie par pays/régions et villes (correspondant approximativement à plus de 50\% du total mondial estimé ; cf. World Gazetteer, 2005). Les écarts différentiels ont été complétés au prorata du poids gravitaire des lieux habités (cf. GEOnet Names Server, données août 2005, plus de 150000 références) pour chaque unité cellulaire ou territoriale représentant une différence par rapport aux recensements/estimations nationaux en 2005.

22. La participation de la population du bassin danubien dans l'ensemble paneuropéen, estimée à $10,4 \%$ en 2005 , est toutefois susceptible de diminuer d'ici 2030 jusqu'à 9,6\% du total(estimation tendancielle par le biais du modèle spatial).

23. Pour assurer l'objectivité optimale du scénario tendanciel appliqué (base croissance de population / PIB, période 2000-2005), les amplitudes très importantes d'accroissement naturel et du PIB ont été rectifiées par les références de la dernière année disponible et par les données de séries temporelles plus longues (cf. Banque mondiale, PIB période 1980-2000).

24. A titre d'exemple, la part relative économique de l'espace danubien (estimée pour 2005 par le modèle spatial) représente un ratio de $7,3 \%$ (très proche des $7,5 \%$ de superficie du total européen), contre au moins $8 \%$ du total estimé à l'horizon 2030.

25. Base de référence : séries annuelles observées depuis plus de 10 ans (Cf. EUROSTAT, Banque mondiale, World Factbook 1995-2005).

26. La croissance tendancielle totalisée pour la période 2005-2030, de l'espace danubien représente $123 \%$ contre $103 \%$ pour l'Europe (projections tendancielles réalisées par l'auteur, à partir du modèle de contiguïté territoriale).

27. La résolution opérationnelle de «raster» de $10 \mathrm{~km}$, voire $1 \mathrm{~km}$ parait encore plus appropriée dans un contexte local, interurbain ou interrégional, comparée à celle utilisée ici (i.e. $25 \times 25 \mathrm{~km}$, échelle paneuropéenne et mondiale).

28. Le choix des facteurs déterminant la cohésion et la convergence interrégionales résulte de l'interconnexion virtuelle spatiale des localités dotées de valeurs supérieures à la moyenne (européenne et/ou danubienne) des indicateurs observés (infrastructures, populations et richesses économiques). 
29. Concrètement ici les extrapolations modélisées (relations de convergence linéaire) cadrées sur l'espace danubien sont générées par les rapports fonctionnels entre les valeurs des PIB en PPA en 1000 USD rapportées par km d'infrastructure multimodale, au niveau de chaque unité spatiale.

30. Dans le contexte du bassin oriental, il s'agit concrètement des relations spatiales réciproques (identifiées par le modèle) entre les ports maritimes/fluviaux et les principaux pôles économiques de la région (capitales et centres régionaux).

31. D'après les sources officielles, la croissance moyenne annuelle du trafic fluvial recensé sur le Danube en 2003 et 2004, de 4-5\% est susceptible de totaliser une croissance totale comprise entre 50\% (hypothèse basse) et plus de 300\% (hypothèse haute), à l'horizon 2015 (cf. Navigation Ports \& Industries, avril 2005).

\section{RÉSUMÉS}

Comment estimer le potentiel, l'évolution et la dynamique spatiale ou temporelle d'un ensemble géographique controversé et (re)composé comme le bassin danubien, lorsque les données statistiques sont incomplètes, peu compatibles et démunies de références spatiales? Afin de répondre à cette problématique complexe et mieux appréhender les mutations en cours, une approche expérimentale, assistée par un modèle spatial original et un système d'information géographique (SIG), est proposée dans l'optique de localiser au moins globalement les interactions entre les infrastructures de transport, les dynamiques démographiques et les richesses économiques, dans le contexte d'une cohésion territoriale européenne à construire et ce dans un double perspective, spatiale et temporelle. Cette analyse à dimension prospective permettra de faire ressortir la place des Balkans dans le système danubien.

\section{INDEX}

Index géographique : Danube

Mots-clés : Transports, Aménagement, Corridors paneuropéens, Population

\section{AUTEUR}

\section{VESELIN SIAROV}

Docteur en Géographie physique, humaine, économique et régionale, Spécialité Aménagement et Transport.siarov@free.fr 